La

Révolution

française

\section{La Révolution française}

Cahiers de l'Institut d'histoire de la Révolution française

$16 \mid 2019$

1801-1840 - Haïti, entre Indépendance et

Restauration

\title{
Antonino de Francesco, La guerre de deux cents ans. Une histoire des histoires de la Révolution française, Paris, Perrin, 2018, 442 pages.
}

Valentine Brunet

\section{OpenEdition}

\section{Journals}

Édition électronique

URL : http://journals.openedition.org/lrf/2912

DOI : $10.4000 /$ Irf.2912

ISSN : 2105-2557

Éditeur

IHMC - Institut d'histoire moderne et contemporaine (UMR 8066)

Référence électronique

Valentine Brunet, «Antonino de Francesco, La guerre de deux cents ans. Une histoire des histoires de la Révolution française, Paris, Perrin, 2018, 442 pages. », La Révolution française [En ligne], 16 | 2019, mis en ligne le 20 juin 2019, consulté le 10 décembre 2020. URL : http://journals.openedition.org/Irf/2912 ; DOI : https://doi.org/10.4000/Irf.2912

Ce document a été généré automatiquement le 10 décembre 2020.

(C) La Révolution française 


\title{
Antonino de Francesco, La guerre de deux cents ans. Une histoire des histoires de la Révolution française, Paris, Perrin, 2018, 442 pages.
}

\author{
Valentine Brunet
}

1 L'histoire de la Révolution française a longtemps soulevé les passions, par l'ampleur des bouleversements qu'elle a entraînés et la portée universelle de son message émancipateur. On lui a souvent opposé l'image mythique d'une "Glorieuse Révolution » d'Angleterre (1688) qui se serait déroulée sans aucune effusion de sang et aurait suscité un large consensus. Cependant, les luttes avaient été tout aussi violentes outreManche, même si elles s'étaient exprimées au cours de la première Révolution de $1642-1660^{1}$. Si 1688 permit de résoudre ces tensions, ce ne fut qu'au prix d'une répression féroce en Irlande. Pourtant, les ruptures révolutionnaires de part et d'autre de la Manche avaient été conduites dans un esprit différent. John Locke, dont les écrits accompagnent 1688, met l'accent sur le pacte fondateur liant les gouvernants et leurs sujets, ce qui justifierait, en cas de félonie du roi, un droit de rébellion du peuple ${ }^{2}$. Mais ce droit à la résistance ne s'exercerait que dans les situations exceptionnelles. Ainsi, le recours à une "révolution» restait conçu comme un moyen de revenir à un point fixe: en 1688, il s'agissait de rétablir la constitution mixte de la monarchie anglaise garantissant un équilibre des pouvoirs entre Roi, Lords et Communes. À l'inverse, c'est la radicalité de 1789 en France qui consacre le sens nouveau du mot "révolution ». Le défi lancé aux monarchies d'Ancien Régime par les héritiers du Contrat social de Rousseau consiste à faire confiance au volontarisme politique en vue de bâtir un régime neuf conforme à des principes abstraits de liberté et de justice.

2 Aussi n'est-il pas étonnant que les défenseurs de l'ancienne société d'ordres se soient rapidement ligués contre la menace grandissante représentée par la Révolution française. Révolution et Contre-Révolution vont d'emblée de pair et ce choc inéluctable marque le début d'une "guerre de deux cents ans ", selon le titre évocateur du livre d'Antonino de 
Francesco. Au fil des combats, c'est bien à la naissance de la catégorie politique de la modernité qu'on assiste. L'auteur rapproche le projet d'autonomie incarné par la Révolution française, posant la société comme créatrice de ses propres normes et valeurs, des «meilleures histoires de la Révolution», qui accompagnent ce processus de démocratisation. Il entend éclairer «la construction de la culture politique des principaux États d'Europe et d'Amérique par l'intermédiaire des fortunes historiographiques de la Révolution » (p. 14-15).

3 Portée aux nues par l'historiographie militante du XIX siècle, vouée aux gémonies par ses adversaires nostalgiques de l'Ancien Régime, la Révolution a joué un rôle essentiel dans l'imaginaire collectif et la vie politique en France. Soulevant le patriotisme, projetant son messianisme sur toute l'Europe, la Révolution est l'événement majeur qui détermine les grandes lignes de fracture politiques au xix ${ }^{e}$ siècle. Dès la Restauration, le débat est vif entre « ultras » et libéraux, et les historiens ne vont cesser d'alimenter les polémiques par leurs interprétations à haute teneur idéologique de la décennie révolutionnaire. Sous le régime de Juillet, le clivage se complexifie, les historiens favorables à la Révolution se séparant en diverses écoles, républicaines, socialistes et communistes. Désormais, ce sont les libéraux qui aspirent à «terminer la Révolution » et défendent la paix à tout prix, comme Guizot et les Doctrinaires ${ }^{3}$, tandis que la gauche patriote veut poursuivre dans la lignée du messianisme révolutionnaire ${ }^{4}$. Malgré l'enracinement progressif du régime républicain après 1875 , la barricade symbolique entre héritiers radicaux et socialistes de la geste révolutionnaire et conservateurs de sensibilité libérale ou monarchiste, n'est jamais vraiment tombée. Ce sont les représentants, éminents ou obscurs, classiques ou originaux, de cette "guerre de deux cents ans » que convoque Antonino de Francesco dans son ouvrage éponyme.

Si la Révolution fait l'objet de débats nourris en France tout au long des $\mathrm{XIX}^{\mathrm{e}}$ et $\mathrm{xx}^{\mathrm{e}}$ siècles, les controverses ne se sont pas limitées au cadre national, loin s'en faut. En cela, la perspective comparative choisie par Antonino de Francesco apparaît particulièrement féconde. En effet, l'historiographie de la Révolution française a déjà été bien étudiée dans le cadre national ${ }^{5}$. La réflexion comparatiste de l'auteur permet de percevoir la Révolution à l'aune des influences réciproques et des échanges entre des traditions historiographiques fort diverses, britannique, italienne, allemande, russe et américaine. Sans être simple, cette tâche est conduite avec brio par l'auteur, qui réussit à faire dialoguer et à montrer les croisements entre les différentes approches nationales, les choix qui sous-tendent les stratégies d'interprétation de l'événement révolutionnaire, l'impact du contexte sur la réception de l'historiographie française à l'étranger.

5 Universaliste et inédite dans son rationalisme politique, la Révolution française a d'abord suscité l'effroi, tout particulièrement outre-Manche. C'est cette radicalité du mouvement révolutionnaire initié en 1789 qui provoque la naissance de l'historiographie contrerévolutionnaire, avec la parution des Reflections on the Revolution in France (novembre 1790) d'Edmund Burke ${ }^{6}$. Dans ce pamphlet sans concessions, le penseur anglo-irlandais pose les fondements de l'argumentation conservatrice, qui condamne la décision des révolutionnaires de faire table rase du passé. Burke reproche à la Constituante de méconnaître l'importance de la "prescription ", de la durée et de l'hérédité, qui seules consacrent les droits historiques et concrets d'un peuple ${ }^{7}$. Son raisonnement oppose les droits concrets des Britanniques, garantis en 1688-1689, aux principes universels des droits de l'homme, proclamés en France en 1789, qu'il considère comme une chimère ${ }^{8}$. On ne saurait créer de nouvelles institutions solides ex nihilo, car un système politique est le 
fruit d'une longue maturation historique, d'un développement graduel, de l'expérience accumulée au fil des siècles. Pour Burke, les droits et libertés doivent être tirés du précédent. Loin d'incarner des valeurs rationnelles, telles la liberté et l'égalité, une constitution est une forme mystérieuse, baroque, dont les ressorts demeurent voilés aux yeux du peuple et dont l'antiquité est le meilleur gage de validité. Burke préfère la sagesse des ancêtres aux idées «dogmatiques» des philosophes français des Lumières? S'il admet la notion de révolution, c'est uniquement en son sens premier, cyclique, visant à rétablir un ordre ancien. Cette idée n'était pas nouvelle : tout au long du XviII ${ }^{\mathrm{e}}$ siècle, les historiens britanniques avaient mis en scène le compromis constitutionnel issu de la "Glorieuse Révolution» de 1688, cette révolution-restauration ${ }^{10}$. Néanmoins, Burke pousse cette logique encore plus loin. Dans son esprit, le respect des traditions est l'une des principales conditions du maintien de la société ; chaque pays possède ses précédents, sa culture, ses préjugés, sans lesquels le tissu social se déchire. D’un traditionalisme, Burke a fait un véritable plaidoyer pour le conservatisme. De Francesco souligne combien ses idées fondamentales résonnent dans le débat public anglais, séduisant aussi bien les historiens que les publicistes. À cet égard, l'auteur aurait pu citer l'œuvre de Walter Bagehot, auteur libéral influent, dont les essais historiques et politiques et les travaux de journaliste reflètent bien l'emprise du « paradigme interprétatif » burkien (p. 122) outreManche au XIX ${ }^{\mathrm{e}}$ siècle $^{11}$.

6 L'auteur montre que l'historiographie britannique se construit dans l'opposition frontale à la Révolution française, accusée de heurter toutes les spécificités nationales, en particulier le parlementarisme modéré et les réformes graduelles. À travers le combat que mène Burke contre la modernité politique, identifiée à la France depuis 1789, se consolide un autre mythe fondateur, celui de l'exceptionnalisme britannique. Ce modèle anglais réformiste est promis à un bel avenir, et ce malgré la tentative de Thomas Paine d'importer le langage révolutionnaire dans son pays natal. Son éloquente réplique à Burke, Rights of Man (1791-1792) ${ }^{12}$, circule dans les milieux du radicalisme populaire, mais ne rencontre que le mépris des élites éduquées. Figure républicaine anglaise, Paine a pris part aux Révolutions américaine et française, jeté des ponts entre les deux rives de l'Atlantique en réactivant le modèle américain sur le continent européen (p. 55). Dès les années révolutionnaires, on voit ainsi se dessiner un creuset républicain commun face à la résistance conservatrice britannique. Des oppositions conceptuelles, appelées à durer, se mettent en place: rupture violente avec l'Ancien Régime contre apparence de continuité institutionnelle; révolution contre réforme graduelle; république étatiste contre monarchie libérale (p. 35-37, p. 65-66, p. 79-81).

7 Ces divergences de fond structurent l'histoire littéraire et politique de la Révolution, qui prend son essor sous la Restauration. C'est tout l'objet du second chapitre du livre, consacré à la confrontation des historiens libéraux, romantiques et socialistes avec un passé révolutionnaire encore vivant. Ces auteurs ont accès à de nombreux témoins ayant survécu à la Terreur et à l'Empire. On peut ainsi distinguer deux types d'historiographie à cette époque : celle rédigée par des survivants de l'ère révolutionnaire, comme JacquesAntoine Dulaure, resté républicain envers et contre tous les changements de régimes et soucieux de prendre position dans les débats politiques contemporains; et celle de la nouvelle école historique française, œuvre de la jeune génération, incarnée par des journalistes engagés comme Adolphe Thiers et François-Auguste Mignet, bientôt acteurs de la Révolution de 1830. 
De Francesco restitue avec brio le caractère vibrant et passionné de la polémique historiographique, qui se développe d'abord contre les velléités de retour en arrière des ultras. Ses acteurs tentent de retrouver le fil des luttes de classes dans un passé que les contre-révolutionnaires présentaient comme idyllique. C'est ainsi qu'Augustin Thierry traite du conflit entre Gaule romanisée et Francs $^{13}$, et cherche à faire revivre " une tradition de liberté que seul l'autoritarisme de l'Ancien Régime, contre lequel les écrivains révolutionnaires s'étaient tellement insurgés, avait réussi à masquer » (p. 76). Le leitmotiv de la lutte des classes est tout aussi déterminant dans l'analyse que fait Guizot des progrès de la civilisation en Europe, à une époque où il est encore le fer de lance de l'opposition libérale aux ultras ${ }^{14}$. Guizot distingue divers éléments en tension permanente dans l'ordre social, sans qu'aucun ne puisse jamais s'imposer complètement aux autres. La lutte des classes fascine cet historien libéral, chantre de la société bourgeoise, qui lui semble le parangon du progrès face à la tyrannie représentée par la monarchie absolue. L'unité nationale est appelée à se construire sous l'égide de ce Tiers état que Guizot appelle "classes moyennes». Mais son idéal s'arrête à 1791, d'où sa fascination pour la révolution «conservatrice » anglaise de 1688, qui rétablit un régime de monarchie mixte et parlementaire. Comme le remarque de Francesco : «Pour Guizot, (...) la référence à imiter se trouvait outre-Manche. En Angleterre, le désastre de la guerre civile de 1640 avait été racheté par la glorieuse révolution parlementaire de 1688, qui avait permis à la société anglaise d'unifier le passé national, tout en perfectionnant en même temps un système de libertés civiles et politiques. » (p. 79) Les yeux tournés vers les rivages britanniques, Guizot s'imaginait ainsi pouvoir construire un autre modèle politique national, fondé sur la Charte et le tournant orléaniste de 1830. Le pays réel n'avait toutefois pas été consulté sur ce tournant et Guizot sera contraint à l'exil londonien, après la révolution de 1848 . De cet opposant libéral, devenu pilier d'un régime conservateur après la révolution de Juillet, il reste néanmoins un legs incontournable, que l'auteur définit comme une passion pour l'histoire et la publication des sources primaires.

9 Cependant, les historiens libéraux eux-mêmes furent dépassés par le succès de la vision déterministe et conflictuelle de la Révolution qu'ils avaient contribué à répandre. $\mathrm{Si}$, selon Thiers et Mignet, la Révolution, violences de la Terreur incluses, était nécessaire pour subvertir l'Ancien Régime et inaugurer la modernité politique, alors pourquoi un nouveau bouleversement ne serait-il pas enclenché par les oubliés ou les frustrés de 1830, prolétaires exclus du suffrage et critiques républicains de la monarchie de Juillet? De Francesco souligne «l'enthousiasme inattendu des nouvelles générations, absolument fascinées par le caractère prédéterminé de l'histoire humaine » (p. 92). De fait, « la grande Révolution redevenait désormais une menace à l'ordre souhaité par Mignet et Thiers » (p. 94).

10 Dès le lendemain de la révolution de Juillet, la flamme de la contestation reprenait de la vigueur. Une école rivale d'historiens se faisait jour, héritiers de la Montagne. Albert Laponneraye ouvrait ainsi un cours public d'histoire de France à l'automne 1831, puis publiait en 1838 son Histoire de la Révolution française, de 1789 jusqu'en $1814^{15}$. Buchez et Roux lançaient une série monumentale d'Histoire parlementaire de la Révolution française, tandis qu'Alphonse Esquiros faisait l'Histoire des Montagnards à la veille de la Révolution de février $1848^{16}$. Mais les admirateurs de 1793 se voyaient débordés par la vague humanitaire et romantique, incarnée, en 1847, par le succès foudroyant de l'Histoire des Girondins d'Alphonse de Lamartine ${ }^{17}$. L'illusion lamartinienne s'effondra brutalement lors 
des sanglantes journées de Juin 1848. Loin de l'aimable présentation offerte par l'auteur des Girondins, Jules Michelet jetait les bases d'une histoire républicaine axée sur le peuple, et solidement étayée par l'usage critique des sources ${ }^{18}$. Malgré leur commune opposition à la dérive conservatrice de la $\mathrm{II}^{\mathrm{e}}$ République, Jules Michelet et Louis Blanc tissent des interprétations divergentes. Blanc célèbre la figure héroïque de Robespierre ${ }^{19}$, qui n'avait été acculé à l'usage de la Terreur que par la «dynamique révolutionnaire» (p. 151). Ces querelles internes au camp républicain étaient en décalage par rapport au véritable ressort caché des explosions révolutionnaires que connaissait la France depuis 1789, selon l'interprétation donnée par Alexis de Tocqueville dans L'Ancien Régime et la Révolution en $1856^{20}$. Tocqueville pointait la responsabilité d'un «égalitarisme forgé par un système centralisateur » (p. 164). C'est un républicain en exil, Edgar Quinet, qui devait parachever la révision en profondeur du paradigme interprétatif de la Grande Révolution, dans un ouvrage intitulé sobrement $L a$ Révolution ${ }^{21}$. Quinet retournait la thèse tocquevillienne de la continuité contre l'influence prolongée, mais néfaste, de l'Église catholique sur les esprits des révolutionnaires, culminant dans la dictature "inquisitoriale » de l'an II (p.172). Cette réflexion sur le lien entre révolution politique et esprit religieux affaiblissait la vision de la Révolution comme point de départ de la modernité politique, en France et dans l'espace européen.

La rechute impériale de la France sonne l'heure de la désillusion envers la Révolution. Ni 1789, ni 1793 ne semblent plus pouvoir servir de points de référence dans la construction de républiques fondées sur des nationalités fières de leur langue, de leur culture et de leurs traditions propres. Le républicanisme italien, sous l'influence de Giuseppe Mazzini, se détourne après le 2 décembre 1851 d'une France "prisonnière de son passé " dictatorial (p.183) pour tenter de tracer une voie démocratique indépendante et patriotique. De même, de Francesco montre que Karl Marx prend ses distances avec la Révolution française après l'échec du printemps des peuples, pour mieux en appeler au dépassement des aventures nationales, la lutte des classes ignorant les frontières. Pourtant, la fascination longtemps exercée sur la gauche européenne par le gouvernement de l'an II devait ressurgir, et inspirer la prise de pouvoir des bolcheviks en octobre 1917 en Russie.

Tandis que la référence à 1793 était mobilisée par l'élan révolutionnaire russe, le monde britannique puisait à nouveau dans l'historiographie conservatrice pour élever des digues contre "la vague révolutionnaire du continent", de peur qu'elle ne "submerge et [n']efface la spécificité culturelle britannique» (p. 276). Le succès rencontré par une autrice anglaise passionnée d'histoire, Nesta $\mathrm{H}$. Webster, est symptomatique de ce retour aux sources burkiennes dans l'entre-deux-guerres ${ }^{22}$. Aux États-Unis, c'est l'œuvre de Crane Brinton qui s'impose comme la plus novatrice. Ancien élève de Harold Laski, qu'il a suivi à Oxford, Brinton approfondit la connaissance du fait révolutionnaire par l'apport de disciplines récentes, telles la sociologie et l'histoire économique. Son livre, The Jacobins. An Essay in the New History, fera date, en dépit d'un accueil plus que réservé d'Albert Mathiez $^{23}$. Brinton récidive en 1934, avec son ouvrage A Decade of Revolution ${ }^{24}$, où il pointe l'incapacité de la classe politique révolutionnaire à maîtriser la situation de crise à partir de 1792, et leur recours à la Terreur comme outil de légitimation du pouvoir. Pour Brinton, Thermidor acquiert dès lors le statut central de retour au dialogue entre le pouvoir politique et la société, ce qui débouche sur une réévaluation positive de l'œuvre du Directoire, vue comme une tentative de stabilisation et de progrès vers la démocratie représentative. 
Entre-temps, l'idée républicaine s'était affirmée en France et la Révolution faisait l'objet de relectures passionnées, par l'historien Alphonse Aulard, dans son Histoire politique de la Révolution française (1901) ${ }^{25}$, et par l'intellectuel et homme politique Jean Jaurès, auteur d'une Histoire socialiste de la Révolution française (1901-1904) ${ }^{26}$, destinée à renouveler durablement les recherches sur les aspects économiques et sociaux de la décennie révolutionnaire. Si l'histoire républicaine d'Aulard a eu des échos importants au-delà des frontières françaises, elle a aussi provoqué des réactions hostiles dans les cercles libéraux et conservateurs, notamment en Allemagne, et s'est heurtée à la méfiance persistante des auteurs socialistes. Dans ces milieux de la gauche militante, l'Histoire de Jaurès connut une postérité féconde. La quête novatrice, lancée par Jaurès sur le terrain socio-économique, a inspiré toute une génération de chercheurs, d'Ernest Labrousse à Albert Mathiez, en passant par Georges Lefebvre. De Francesco rend un très bel hommage aux travaux de Lefebvre, qui dominent l'histoire révolutionnaire dans les années 1930. Il se montre plus sceptique quant au raidissement «dogmatique » de Lefebvre à partir du second aprèsguerre (p. 314). Mais l'essentiel est ailleurs, dans l'institutionnalisation réussie des études révolutionnaires en France. Chargé de préparer les célébrations du $150^{\mathrm{e}}$ anniversaire de la Convocation des États-Généraux, Lefebvre obtient la création d'un Institut d'Histoire de la Révolution française par un décret du ministre de l'Instruction, Jean Zay, en 1937. Auparavant, il avait ouvert un certain nombre de chantiers majeurs en histoire économique de la Révolution. Ces projets de recherche s'inscrivaient dans le bouillonnement intellectuel de la préparation $\mathrm{du} 150^{\mathrm{e}}$ anniversaire et reçurent une nouvelle impulsion de la victoire du Front populaire. Dans un contexte de mouvement social d'une ampleur sans précédent, la Grande Révolution prenait une résonance particulière. Néanmoins, la guerre menaçait et ce n'est qu'une célébration en demi-teinte qui pourra se tenir, en dépit des efforts de Lefebvre. Celui-ci ne cessera d'enseigner durant la Seconde Guerre mondiale et assurera ainsi la pérennité de la chaire d'Histoire de la Révolution française. C'est cette "volonté de sauvegarder la tradition de la France révolutionnaire et universitaire»(p.311) qui a toujours animé Lefebvre, comme en témoigne son intense activité en tant que directeur de l'Institut d'Histoire de la Révolution française jusqu'à sa mort en $1959^{27}$.

Après 1945, la présence très marquée du modèle marxiste chez Lefebvre, parfaitement assumée, constitue à la fois le socle d'une école internationale de chercheurs intéressés par la problématique des mouvements populaires et un repoussoir pour certains historiens moins engagés sur le plan idéologique. De Francesco discerne une lente mutation qui s'opère parmi certains anciens élèves de Lefebvre, comme Richard Cobb, historien britannique exerçant à Oxford ${ }^{28}$. Cobb approfondit la piste d'une histoire empirique de la Révolution qui se concentre sur l'impact concret du bouleversement révolutionnaire dans la vie quotidienne et tente d'élucider la réaction des groupes les moins étudiés, notamment en province, à la politique jacobine ${ }^{29}$.

En 1978, toutefois, un véritable tournant se produit dans l'historiographie de la Révolution avec la parution de l'opus de François Furet, Penser la Révolution française ${ }^{30}$. La présentation qu'en donne de Francesco est ambiguë : s'il reconnait que Furet a encouragé les historiens, notamment anglo-saxons, dans la voie d'un révisionnisme aux accents conservateurs, il considère dans le même temps que « c'est uniquement grâce à Furet que l'historiographie de la Révolution a perdu un trait messianique qui l'avait longtemps accompagnée et que, après son intervention, rien n'a plus été comme avant en matière d'études révolutionnaires » (p. 342). Il est difficile de suivre de Francesco lorsqu'il salue la 
"grande originalité»(p.338) de la contribution de Furet à l'historiographie de la Révolution. En effet, la voie révisionniste empruntée par Furet avait été toute tracée par la conférence fameuse d'Alfred Cobban, prononcée le 6 mai 1954 à l'University College London et intitulée "The Myth of the French Revolution $»^{31}$. L'historien britannique prônait déjà l'inscription de la "révolution » française dans la longue durée, persuadé qu'il n'y avait pas de rupture nette avec la féodalité et encore moins naissance d'une société bourgeoise. Or, de Cobban à Furet, la thèse d'une révolution qui n'aurait servi à rien semble toujours aussi faible, comme l'a montré Eric Hobsbawm: "[c'est] le type même de proposition contrefactuelle qui ne peut ni être testée, ni revêtir la moindre plausibilités ${ }^{2}$.» Si la Révolution n'avait été qu'une simple péripétie, si elle n'avait guère modifié la physionomie de la société française du XIX siècle, qui devait de toute façon émerger des tendances lourdes déjà à l'œuvre sous l'Ancien Régime, pourquoi les libéraux de la Restauration auraient-ils défendu la Révolution, toute la Révolution, et proclamé son rôle historique incontournable dans le progrès des libertés ${ }^{33}$ ? À force de prendre de la «distance avec l'événement qui avait toujours accompagné la politique française » (p. 342), on peut se demander si Furet n'a pas perdu de vue l'essentiel, c'est-à-dire la différence entre les tendances de fond agissant dans une société donnée et la difficulté d'éliminer le joug féodal autrement que par une secousse révolutionnaire majeure. Il est dommage que de Francesco n'ait pas explicité la dette de Furet envers Cobban, ni indiqué les limites de l'entreprise révisionniste. L'auteur préfère insister sur l'audace d'un Furet "expiant son passé communiste» à travers ses attaques répétées contre les interprétations d'inspiration marxiste (p. 342). Sans nier l'importance du renouvellement de l'historiographie révolutionnaire classique, l'approche révisionniste s'est parfois égarée dans les limbes de l'histoire conceptuelle, tant et si bien que le tournant furetien a vite été dépassé par une autre vague, venue d'outre-Atlantique.

C'est à travers l'étude du lien entre les Révolutions d'Amérique et de France que le renouvellement de l'histoire révolutionnaire allait s'imposer. Dépassant la notion $\mathrm{d}^{\prime}$ ' exception française ", chère à Michel Vovelle ${ }^{34}$, des chercheurs ont avancé l'hypothèse selon laquelle la décennie 1789-1799 s'inscrirait dans un mouvement plus large de révoltes et de troubles révolutionnaires de part et d'autre de l'Atlantique. Au XVIII ${ }^{e}$ siècle, cet espace atlantique était devenu relativement homogène grâce à l'explosion des échanges démographiques, culturels, épistolaires et commerciaux. Loin d'être limitées à la France, les Lumières avaient une dimension transnationale, voire cosmopolite. Des travaux précurseurs de Jacques Godechot, pour la partie française, et de Robert Palmer, pour l'aire américaine, présentés au Congrès international des sciences historiques de Rome en 1955, ont été un temps entravés par le contexte de guerre froide ${ }^{35}$. Pourtant, faisant fi d'un exceptionnalisme américain aux accents souvent conservateurs, Robert Palmer publie entre 1959 et 1964 son étude majeure en deux volumes, The Age of the Democratic Revolution ${ }^{36}$, où il élabore la thèse d'une "révolution atlantique », mère de la démocratie moderne, ayant commencé en Amérique du Nord vers 1770, rebondi en France dans les années 1780, puis secoué tout le Vieux Continent. Comme le souligne de Francesco, « cette période de tensions finit par prendre la forme d'un formidable défi à l'égard du privilège, c'est-à-dire envers toutes ces élites qui utilisaient les rentes de position garanties par le privilège comme un instrument de domination.» (p. 348) La formule condense, en des termes très éclairants, les recherches de Palmer, reprises et développées en France par Jacques Godechot ${ }^{37}$. Ces travaux ont mis en évidence l'existence d'une culture révolutionnaire et républicaine commune, véritable terreau des 
crises politiques de la fin du XVIII ${ }^{e}$ siècle. Plus récemment, Annie Jourdan a repris ce dossier des "révolutions atlantiques », en insistant sur l'influence du modèle américain en France, qui a éclipsé le système de monarchie mixte à l'anglaise, auparavant très admiré. La Révolution américaine a inspiré la Déclaration des droits de 1789. Certes, les Constituants ont ensuite voulu surpasser le modèle d'outre-Atlantique; deux approches concurrentes se sont alors partagé le flambeau de la révolution, et toutes deux ont eu un fort retentissement dans l'Europe de la fin du XVIII ${ }^{\mathrm{e}}$ et du début du XIX ${ }^{\mathrm{e}}$ siècle ${ }^{38}$.

Grâce à ces travaux, la page des histoires purement nationales de la Révolution paraît tournée. Ce constat ne semble pas réjouir de Francesco, qui craint la marginalisation de la Révolution française comme objet historique central. Ce pessimisme n'est pas nouveau: Claude Mazauric déplorait déjà, il y a près de quinze ans, que «la Révolution française cesse de miroiter au centre de l'entreprise de sémiologie de l'histoire d'où elle a rayonné si longtemps ${ }^{39}$.» Pourtant, comparer, mettre en perspective, l'événement fondateur de la modernité politique, est-ce nécessairement le marginaliser ? Cette confrontation avec les autres révolutions de la fin $\mathrm{du} \mathrm{XvIII}^{\mathrm{e}}$ siècle dans l'espace atlantique n'est-elle pas, au contraire, une source d'enrichissement du prisme interprétatif ? Il convient de nuancer l'image sombre que projette l'épilogue du livre, intitulé «Les cendres de la Révolution ». Replacer la Révolution française au sein d'un espace plus vaste, ce n'est pas nier l'importance, l'impact et les caractéristiques singulières de la décennie 1789-1799, c'est sortir du francocentrisme, en reconnaissant ce que les idées des révolutionnaires français doivent aux mouvements qui les ont précédés, en Angleterre, aux Pays-Bas et aux ÉtatsUnis $^{40}$. Certes, le culte mémoriel a, dans une certaine mesure, remplacé une véritable vision historique de la décennie révolutionnaire. Mais les pistes innovantes de recherche ne manquent pas, et sont soulignées par l'auteur lui-même, lorsqu'il évoque le chantier du Directoire, relancé au début des années 2000 par des historiens tenaces tels que Bernard Gainot et Pierre Serna ${ }^{41}$. L'essor d'une nouvelle culture transnationale dans les études révolutionnaires peut être perçu comme un signe de renaissance, et non comme la menace d'une submersion de la Révolution française par l'histoire globale. L'ouvrage d'Antonino de Francesco, où se mêlent tant de références historiographiques de pays différents, témoigne non seulement de l'immense savoir de son auteur, mais aussi de sa capacité à s'approprier ce contexte de plus en plus international. Le succès de l'histoire de la Révolution française dépend des nouveaux prolongements que permet justement l'élargissement du cadre culturel et géographique. Cet horizon promet un bel avenir aux recherches révolutionnaires et il est certain que l'ouvrage d'Antonino de Francesco offre une riche base de travail aux historiens qui s'engagent dans cette direction. Avoir mis son excellente connaissance de l'historiographie révolutionnaire au service d'un public varié, des érudits aux étudiants, dans une synthèse claire et complète, mérite toute notre reconnaissance. 


\section{NOTES}

1. Pierre SERNA, «Les deux corps du mythe révolutionnaire ", in D. Kalifa (dir.), Les historiens croient-ils aux mythes? Paris, Publications de la Sorbonne, 2016, p. 217-236, spéc. p. 219.

2. Le Second Traité du gouvernement civil paraît en 1690, même s'il a été rédigé antérieurement; John LOCKE, Two Treatises of Government, éd. Peter Laslett, Cambridge, Cambridge University Press, 1988 ; trad. de l'anglais, avec introduction et notes par Jean-Fabien Spitz, Le second traité du gouvernement, Paris, PUF, 1994.

3. Servane MARzIN, "L'Europe de François Guizot: le dernier échec? " in François Guizot (1787-1874) : Passé - Présent, Actes du Colloque de la Société d'Histoire Moderne et Contemporaine de Nîmes et du Gard, 20 - 22 novembre 2008, Paris, L'Harmattan, 2010, p. 319-331.

4. Philippe DARRIUlAT, Les Patriotes. La gauche républicaine et la nation, 1830-1870, Paris, Le Seuil, coll. «L'univers historique », 2001.

5. Cf. notamment l'ouvrage d'Olivier BÉTOURnÉ et Aglaia I. HARTIG, Penser l'histoire de la Révolution. Deux siècles de passion française, Paris, La Découverte, coll. " Armillaire », 1989, et le livre, déjà ancien mais toujours instructif, d'Alice GÉRARD, La Révolution française: mythes et interprétations, 1789-1970, Paris, Flammarion, coll. « Questions d'Histoire », 1970.

6. Edmund BURKE, Reflections on the Revolution in France (1790), éd. Frank M. Turner, New Haven, Yale University Press, 2003.

7. «Pour l'Assemblée Nationale de France, la possession n'est rien, le droit et les usages ne sont rien. Je vois l'Assemblée Nationale réprouver ouvertement la doctrine de la prescription. » (cette traduction et celle de la note suivante sont de l'autrice), ibid., p. 127-128.

8. «... cette fiction monstrueuse qui, en inspirant de fausses idées et de vaines attentes aux hommes destinés à marcher dans la voie obscure d'une vie laborieuse, ne sert qu'à aggraver et à rendre plus amère la véritable inégalité qu'elle ne pourra jamais supprimer. », ibid., p. 32.

9. Ibid., p. 76.

10. Jean-Numa DUCANGE, La Révolution française et l'histoire du monde. Deux siècles de débats historiques et politiques, 1815-1991, Paris, A. Colin, 2014, p. 43.

11. The Collected Works of Walter Bagehot, éd. Norman St John-Stevas, Londres, The Economist, 1965-1986, 15 volumes.

12. Thomas PAINE, Rights of Man, Part I, Part II, Londres, J. S. Jordan, 1791-1792.

13. Augustin THIERRY, Dix ans d'études historiques, Paris, J. Tessier, 1835 ; Claude NICOLET, La fabrique d'une nation : la France entre Rome et les Germains, Paris, Perrin, « Pour l'histoire », 2003.

14. François GUIzoT, Histoire de la civilisation en Europe, depuis la chute de l'Empire romain jusqu'à la Révolution française (1828-1829), ré-éd. Pierre Rosanvallon, Paris, Hachette - Pluriel, 1985.

15. Albert LAPONNERAYE, Histoire de la Révolution française, de 1789 jusqu'en 1814, Paris, chez l'éditeur, 1838.

16. Philippe-Joseph BUCHEZ, Pierre-Célestin ROUX-LAVERGNE, Histoire parlementaire de la Révolution française ou Journal des assemblées nationales depuis 1789 jusqu'en 1815, Paris, Paulin, 1834-1838, 40 volumes ; Alphonse ESQUIROs, Histoire des Montagnards, Paris, V. Lecou, 1847.

17. Alphonse DE LAMARTINE, Histoire des Girondins, Paris, Furne et Coquebert, 1847.

18. Jules MICHELET, Histoire de la Révolution française, Paris, Chamerot, 1847-1853, 6 volumes.

19. Louis BLANC, Histoire de la Révolution française, Paris, Langlois et Leclercq, 1847-1862, 10 volumes.

20. Alexis DE TOCQUeVILLE, L'Ancien Régime et la Révolution, Paris, Michel Lévy frères, 1856. 
21. Edgar QUINET, La Révolution, Paris, Lacroix, 1865.

22. Nesta H. WEBSTER, The French Revolution. A Study in Democracy, Londres, Constable and Co., 1919.

23. Crane BRINTON, The Jacobins. An Essay in the New History, New York, Macmillan, 1930.

24. Crane BRINTON, A Decade of Revolution, New York, Harper, 1934.

25. Alphonse AULARD, Histoire politique de la Révolution française, Paris, A. Colin, 1901.

26. Jean JAURÈs, Histoire socialiste de la Révolution française, Paris, J. Rouff, 1901-1904.

27. Pierre SERNA, «Lefebvre au travail, le travail de Georges Lefebvre : un océan d'érudition sans continent Liberté ? », La Révolution française, 2 (2010), [En ligne], consultée le 15 novembre 2018.

28. Voir Richard Совв, A Second Identity. Essays on France and French History, Oxford University Press, 1969.

29. Richard СОВв, The Police and the People. French Popular Protest, 1789-1820, Oxford, Clarendon Press, 1970 ; The French and their Revolution. Selected Writings, éd. David Gilmour, New York, The New Press, 1998 ; La mort est dans Paris. Enquête sur le suicide et la mort violente dans le petit peuple parisien au lendemain de la Terreur, traduit de l'anglais par Daniel Alibert-Kouraguine, intr. Pierre Serna, Anacharsis, 2018.

30. François FURET, Penser la Révolution française, Paris, Gallimard, "Bibliothèque des Histoires ", 1978.

31. Voir Pierre SERNA, «Les deux corps du mythe révolutionnaire » in Dominique Kalifa (dir.), Les historiens croient-ils aux mythes? Paris, Publications de la Sorbonne, 2016, p. 217-236.

32. Eric J. новьвашм, Echoes of the Marseillaise, Two Centuries Look Back on the French Revolution, Londres, Verso, 1990, p. 24 (traduction de l'autrice).

33. Ibid., p. 27.

34. Michel vovelle (dir.), Révolution et République. L'exception française, Actes du Colloque des 21-26 sept. 1992, Université de Paris I, Paris, Kimé, 1994.

35. Jacques GODECHOT, Robert R. Palmer, "Le Problème de l'Atlantique du XVIII ${ }^{\mathrm{e}}$ au $\mathrm{XX}^{\mathrm{e}}$ siècle ", $X$ Congresso internazionale di scienze storiche, vol. V : Storia contemporanea, Florence, Sansoni, 1955, p. $175-239$.

36. Robert R. PALMER, The Age of the Democratic Revolution: A Political History of Europe and America, Princeton (N. J.), Princeton University Press, 1959-1964, 2 vol.

37. Jacques GoDEchot, Les Révolutions, 1770-1799, Paris, PUF, $2^{\mathrm{e}}$ éd., 1965.

38. Annie JOURDAN, La révolution, une exception française? Paris, Flammarion-Champs, 2004, p. 353-374.

39. Claude MAZAURIC, «Traditions et tendances dans l'historiographie de la Révolution française » in Philippe Bourdin, Jean-Luc Chappey (dir.), Révoltes et révolutions en Europe (Russie incluse) et aux Amériques de 1773 à 1802, Paris, CNED-SEDES, 2004, p. 42.

40. A. JOURDAN, op. cit., p. 365.

41. Bernard GAINOT, 1799, un nouveau jacobinisme? La démocratie représentative, une alternative à Brumaire, Paris, Éd. du CTHS, 2001 ; Pierre Serna, La République des girouettes, 1789-1815, et au-delà : une anomalie politique, la France de l'extrême-centre, Seyssel, Champ Vallon, 2005. 


\section{AUTEUR}

\section{VALENTINE BRUNET}

Doctorante IHRF-IHMC

Université Paris 1 Panthéon-Sorbonne 